\title{
Aproximación al método CDC para determinar susceptibilidad a insecticidas en vectores de leishmaniasis
}

\author{
Erika Santamaría ${ }^{1}$, Leonard E. Munstermann ${ }^{2}$, Cristina Ferro ${ }^{1}$ \\ ${ }^{1}$ Laboratorio de Entomología, Instituto Nacional de Salud, Bogotá, D.C., Colombia. \\ 2 Epidemiology and Public Health Departament, School of Medicine, Yale University, New Haven, CT, U.S.A. \\ El Centro de Control de Enfermedades (CDC, EE.UU.) ha propuesto un método simplificado \\ para la determinación de resistencia a insecticidas en mosquitos adultos. El método consiste \\ en impregnar botellas Wheaton de $250 \mathrm{ml}$ con soluciones de insecticida en acetona, transferir \\ grupos de insectos y registrar la mortalidad a intervalos de tiempo regulares por una hora. En \\ la estandarización del método para uso con flebotomíneos, se evaluaron inicialmente botellas \\ controles sin insecticida y posteriormente impregnadas con lambdacihalotrina. Se emplearon \\ hembras de dos colonias de laboratorio: Lutzomyia longipalpis $\left(\mathrm{F}_{50}\right.$ y $\left.\mathrm{F}_{54}\right)$ y Lutzomyia serrana \\ $\left(F_{17}\right)$ y hembras silvestres de Lutzomyia quasitownsendi. Grupos de 10 a 24 hembras, sin \\ alimento sanguíneo, de 1 a 3 días de edad, se transfirieron durante una hora a botellas con \\ los siguientes tratamientos: 1) sin aditivos, 2) 0,5 ml de acetona y 3) 1,0 $\mathrm{ml}$ de acetona. Se \\ realizaron de tres a cinco réplicas para cada tratamiento y especie. En las botellas limpias, \\ sin aditivos, los insectos permanecieron en posición de reposo. En botellas con 0,5 y 1,0 ml \\ de acetona se observó un efecto repelente en L. longipalpis y L. serrana en los primeros 10 \\ minutos de exposición. En una pequeña proporción de hembras de L. serrana se observó \\ postración seguida por una rápida recuperación al mover la botella.La pruebas en campo \\ realizadas con $L$. quasitownsendi produjeron resultados similares a los obtenidos con $L$. \\ serrana. Los ensayos preliminares con insecticida se realizaron con L. longipalpis $\left(\mathrm{F}_{54}\right)$ a 3 \\ concentraciones de lambdacihalotrina (10, 50 y $100 \mu \mathrm{g} /$ botella) y se registraron datos de \\ mortalidad en periodos de 5 minutos; las líneas de regresión para cada concentración se \\ compararon y analizaron en el contexto del método CDC. Las ventajas de este método frente \\ al convencional, en esta evaluación preliminar, fueron: su bajo costo, fácil uso en campo, \\ superficie de exposición totalmente impregnada y visibilidad del comportamiento de los \\ insectos.
}

Palabras clave: susceptibilidad a insecticidas, ensayos biológicos, flebótomos.

\section{Determination of insecticide susceptibility in leishmaniasis vectors by the CDC method}

The Centers for Disease Control (CDC, USA) has proposed a simplified method for the determinations of insecticide resistance in adult mosquitoes, using $250 \mathrm{ml}$ Wheaton bottles containing measured dosages. Insects are transferred into the bottle for 1 hour and monitored for mortality at regular intervals. In standardizing the CDC method for use with phlebotomine sand flies, effects of the solvent without insecticide were evaluated. Two colonized sand fly vector species were used: Lutzomyia longipalpis $\left(\mathrm{F}_{50}\right.$ and $\left.\mathrm{F}_{54}\right)$ and Lutzomyia serrana $\left(\mathrm{F}_{17}\right)$. Groups of 10 to 24 unfed females 1-3 days old were transferred for $1 \mathrm{~h}$ to Wheaton bottles with the following pretreatment: (1) without additive, (2) $0.5 \mathrm{ml}$ of acetone, or (3) $1.0 \mathrm{ml}$ of acetone. Three to 5 replicates were undertaken for each condition and each species. In the control bottles, the insects rested quietly and after $1 \mathrm{~h}$ appeared normal. In bottles with 0.5 and $1.0 \mathrm{ml}$ acetone, a repellent effect was observed in $L$. longipalpis and $L$. serrana within the first $10 \mathrm{~min}$. A small proportion of the L.serrana became prostrate, but recovered quickly after removal from the bottle. Field test performed with Lutzomyia quasitownsendi produced results similar to those of the L.serrana colony flies. The insecticide bioassays were performed with L. longipalpis $\left(F_{50}\right)$ flies. Females were exposed to three graded doses of lambdacyhalothrin $(10,50$ and 100 $\mu \mathrm{g} / \mathrm{bottle})$, and mortality was recorded at five-minute intervals. Regression lines for the 3 con- 
centrations were compared within the context of the CDC method. The advantages of the CDC method over the WHO protocols were four: lower cost, fewer insects required, an entire group of insects exposed to the same surface, and ease of field use.

Key words: Insecticide susceptibility, bioassays, phlebotomine sand flies.

Durante las últimas décadas, la resistencia a insecticidas continúa siendo el problema técnico más importante que afecta los programas de control de insectos vectores y plagas en agricultura, medicina veterinaria y salud pública (1). La Organización Mundial de la Salud (OMS) establece que, aproximadamente, el $40 \%$ de las especies de insectos de importancia médica han sido detectados con algún grado de resistencia a insecticidas (2). Por ello, la determinación y vigilancia periódica de la susceptibilidad a insecticidas debe ser un importante componente en cualquier programa de control químico de vectores.

El método actual para detectar susceptibilidadresistencia a insecticidas en mosquitos adultos a través de ensayos biológicos es el propuesto por la OMS (3) y es el método estándar utilizado desde la década de los 60 . El procedimiento y estuche de materiales es el mismo para mosquitos (géneros Aedes, Anopheles y Culex), flebótomos, simúlidos y ceratopogónidos.

Sin embargo, este procedimiento presenta algunas inconveniencias como son su elevado costo, debido principalmente al envío, disponibilidad de papeles impregnados, dosis diagnósticas inadecuadas para algunos vectores y requerimiento de una cantidad considerable de ejemplares para establecer líneas base susceptibles (4). En la experiencia personal en el uso del estuche OMS, se han observado, además, dos deficiencias; por un lado, se presentan escapes de insectos pequeños, como son los flebótomos, y quizás el más importante defecto es que no toda la superficie del recipiente de exposición está impregnada, ya que el papel impregnado con el insecticida sólo cubre las

Correspondencia:

Cristina Ferro, Grupo de Entomología, Instituto Nacional de Salud.

mferro@ins.gov.co

Recibido: 08/10/02; aceptado: 03/03/03 paredes del cilindro y no las bases superior e inferior del mismo, por lo cual los insectos reciben cantidades diferentes del insecticida, lo que induce a errores significativos en los resultados.

La OMS reconoce también las deficiencias de sus procedimientos estándar y entre las medidas planteadas para solucionar el problema ha recomendado perfeccionar los métodos actuales para detectar resistencia a insecticidas y mejorar su uso en campo. Promueve, también, el desarrollo de métodos alternativos para determinar la resistencia en forma eficaz y práctica (5).

Recientemente, investigadores del CDC (Centers for Disease Control and Prevention) de Atlanta han rescatado una antigua técnica para simplificar la determinación de susceptibilidad o resistencia a insecticidas en mosquitos adultos. Dicha técnica consiste en utilizar un recipiente de vidrio como superficie impregnada y medir el tiempo necesario para que un grupo de insectos incorpore el insecticida y evidencie signos de envenenamiento (6).

Con el fin de observar la conveniencia del método CDC en insectos vectores de leishmaniasis, se plantearon los siguientes objetivos: 1) observar el comportamiento y registrar la mortalidad de tres especies de Lutzomyia en botellas controles sin insecticida, y 2) determinar, en ensayos biológicos de laboratorio, la concentración adecuada de lambdacihalotrina para la especie Lutzomyia longipalpis (Lutz y Neiva 1912).

\section{Materiales y métodos \\ Descripción del método CDC}

Brevemente, la técnica consiste en tratar botellas de vidrio Wheaton de $250 \mathrm{ml}$ de capacidad y superficie interna aproximada de $250 \mathrm{~cm}^{2}$, adicionando una cantidad de acetona suficiente para impregnar la botella y la tapa, en este ensayo 0,5 y $1,0 \mathrm{ml}$, más una cantidad apropiada del insecticida grado técnico, previamente preparado en soluciones stock. Para botellas controles, se 
adiciona únicamente acetona. Se tapa herméticamente la botella y se hace girar la solución en todas las direcciones. Se retira la tapa y se continúa rodando hasta que el solvente se evapora por completo; posteriormente se deja secar sin la tapa por espacio de seis horas. Siguiendo este procedimiento se presume que la botella queda uniformemente impregnada.

Pasado el tiempo de secado, se introducen todos los insectos a la vez y se registra el número de insectos muertos en periodos de tiempo definidos, hasta que la mortalidad sea del $100 \%$.

Para decidir qué concentración de insecticida se debe usar es necesario correr ensayos con botellas impregnadas a diferentes concentraciones de insecticida con grupos de insectos de una población susceptible; de esta forma se pueden determinar dos importantes parámetros: el punto de saturación de insectos susceptibles para un insecticida específico y el tiempo y concentración que puede diferenciar una población susceptible de una resistente (7).

\section{Ensayos con botellas controles}

El primer paso en la aproximación a este método fue valorar el comportamiento de hembras de Lutzomyia dentro de botellas sin insecticida. Se emplearon hembras de 1-3 días de nacidas de dos colonias de laboratorio: L. longipalpis $\left(F_{50}\right)$ y L. serrana (Damasceno y Arouck 1949) $\left(F_{17}\right)$. Las hembras no habían sido alimentadas con sangre, pues la mayoría de estudios de susceptibilidad a insecticidas en flebótomos se utilizan hembras sin alimento sanguíneo, ya que se presenta mayor mortalidad en hembras alimentadas (8-12). Se transfirieron grupos de 10 a 24 hembras durante una hora a botellas con los siguientes tratamientos: (1) sin ningún aditivo, (2) impregnadas con 0,5 ml de acetona, y (3) impregnadas con $1,0 \mathrm{ml}$ de acetona. Se registraron observaciones cada 5 minutos y se realizaron de 3 a 5 réplicas de cada tratamiento para cada especie. Adicionalmente, se efectuaron ensayos preliminares en campo en un foco de leishmaniasis cutánea en Bituima (Cundinamarca) con L. quasitownsendi Osorno, Osorno-Mesa \& Morales, 1972, el flebótomo más abundante en esta zona (datos no publicados, Unidad de Entomología, Laboratorio de Salud
Pública de Cundinamarca), para los cuales se emplearon botellas limpias e impregnadas con 1,0 $\mathrm{ml}$ de acetona.

\section{Ajuste de una concentración adecuada de lambdacihalotrina para L. longipalpis}

Las hembras de L. longipalpis empleadas tanto para los ensayos con botellas controles, como para el ajuste de la concentración de lambdacihalotrina, provenían de una colonia de laboratorio establecida desde 1994 con ejemplares provenientes de Ricaurte, Cundinamarca, sin presión de selección a insecticidas y sin adición posterior de ejemplares silvestres. Para los ensayos con lambdacihalotrina se emplearon hembras de la generación filial 54 .

Se impregnaron botellas con tres concentraciones de lambdacihalotrina grado técnico (Zeneca) (pureza: $91,8 \%$ ): $10 \mu \mathrm{g} /$ botella, $50 \mu \mathrm{g} /$ botella y 100 $\mu \mathrm{g} / \mathrm{botella}$.

Se corrieron ensayos simultáneamente con las tres concentraciones más una botella control (impregnada con $1,0 \mathrm{ml}$ de acetona), se adicionaron grupos de 10 hembras, introduciendo todos los insectos a la vez y se registró el número de hembras muertas en periodos de 5 minutos, hasta que la mortalidad fue del $100 \%$. El criterio de muerte es definido por cada autor. En este estudio, las hembras caídas que no reaccionaban ante un movimiento suave de la botella se consideraron como muertas. Se realizaron tres réplicas para cada concentración. Se elaboró una gráfica con el tiempo vs. el porcentaje de mortalidad y los datos se introdujeron en un análisis de regresión probit usando un programa básico (EPA análisis, versión 1.5).

\section{Resultados}

En las botellas controles limpias, sin aditivos, las hembras de las tres especies permanecieron en posición de reposo, principalmente en el fondo de la botella y su estado, pasada una hora, fue normal, es decir, fueron viables para cría o para otros ensayos.

En el caso de botellas impregnadas con 0,5 y 1,0 $\mathrm{ml}$ de acetona, se presentaron variaciones entre especies: para L. longipalpis se observó repelencia hacia las paredes de la botella en los 10 primeros 
minutos de exposición, después de lo cual, las hembras permanecieron en el fondo en posición de reposo. No se observaron en esta especie hembras caídas durante el tiempo de exposición $y$, pasada una hora, el estado de las hembras fue normal. Para $L$. serrana y $L$. quasitownsendi se observó también repelencia en los primeros minutos de exposición y postración de algunas hembras $(20 \%-30 \%)$, las cuales, después de algunos segundos, se recuperaron nuevamente. Es claro que la acetona, usada como solvente, no se evapora en su totalidad y que los residuos que permanecen en las paredes de la botella producen irritabilidad en algún grado en los flebótomos ensayados.

Se decidió, entonces, iniciar los ensayos con insecticida utilizando $L$. longipalpis, ya que los controles con acetona se comportaron normalmente en esta especie, mientras en las otras dos especies, $L$. serrana y $L$. quasitownsendi, la acción de la acetona produce un efecto de postración-recuperación que, aunque en una proporción baja, podría confundirse con la acción del insecticida y generar errores al registrar los datos de mortalidad.

En los ensayos que incluyeron insecticida, las hembras de L. longipalpis se expusieron continuamente a un contacto tarsal con lambdacihalotrina en tres botellas impregnadas a diferentes concentraciones. Se observó en estos ensayos que al incrementar la cantidad de insecticida, las hembras caían en un menor tiempo. De igual forma, se observó que antes de los cinco primeros minutos no se presentaron hembras caídas. Para las tres concentraciones de insecticida, las primeras hembras empezaron a caer después de los siete minutos de iniciada la exposición. Al parecer este es el tiempo mínimo de incubación del insecticida dentro del insecto, es decir, el tiempo que tarda el insecticida en atravesar la cutícula, ser transportado por la hemolinfa y actuar en las membranas nerviosas produciendo el efecto knock-down, parálisis y posteriormente la muerte (13).

La figura 1 muestra las líneas de regresión tiempomortalidad para las tres concentraciones de insecticida. Entre las concentraciones probadas, la más apropiada parece ser $10 \mu \mathrm{g} / \mathrm{botella}$, ya que

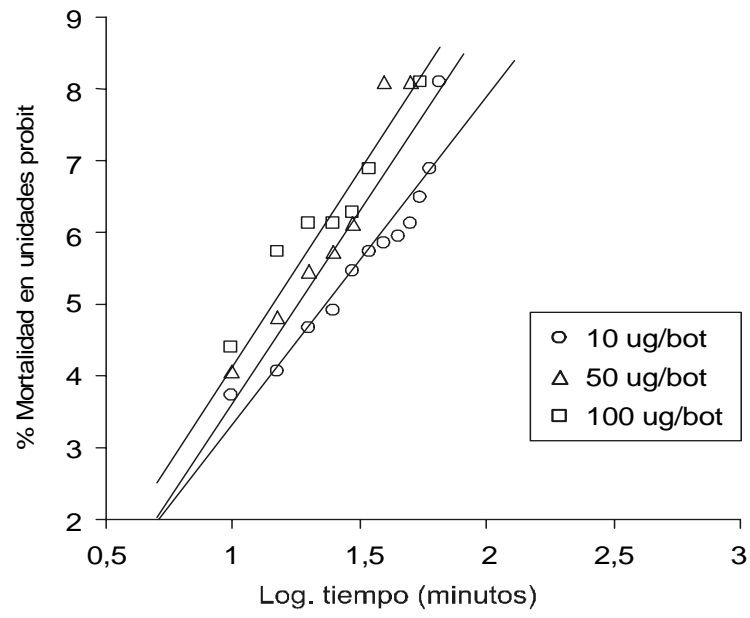

Figura 1. Tendencia de los datos tiempo-mortalidad, en hembras de L. longipalpis expuestas a botellas tratadas con tres concentraciones de lambdacihalotrina. $n=10$ hembras/ réplica, 3 réplicas por concentración, $\mathrm{DE}<5 \%$.

Cuadro 1. Tiempos letales para lambdacihalotrina $(10 \mu \mathrm{g} /$ botella) en L. longipalpis.

\begin{tabular}{|c|c|c|c|c|}
\hline $\mathrm{TL}_{50}{ }^{1}$ & $\mathrm{IC}^{2}$ & $\mathrm{TL}_{95}$ & IC & $\mathrm{m} \pm \mathrm{DS}^{3}$ \\
\hline 24 & $21-26$ & 60 & $53-72$ & $4,12 \pm 0,37$ \\
\hline
\end{tabular}

mata todos los individuos en una hora aproximadamente y produce una línea de regresión que puede ser comparada con las que se generen de otras poblaciones de la misma especie (6). Al introducir en el programa estadístico los datos tiempo-mortalidad registrados a esta concentración (10 $\mu \mathrm{g} /$ botella), se obtuvieron los tiempos letales $\mathrm{TL}_{50}$ y $\mathrm{TL}_{95}$ (cuadro 1). Estos datos y sus intervalos de confianza son el punto de referencia al comparar esta cepa con poblaciones silvestres de L. longipalpis.

\section{Discusión}

En los primeros estudios de los efectos de sustancias insecticidas sobre artrópodos, uno de los métodos empleados determinaba el tiempo en el cual aparecían signos irreversibles de envenenamiento en el $50 \%$ de los individuos expuestos. Este método, usado por algunos 
investigadores en la década de los 40 (14), no registraba mortalidad, sólo el tiempo que tomaban individuos permanentemente expuestos en ser derribados (KT: knock-down time). Más tarde, surgieron otros métodos que calculaban dosis letales $\left(L_{50}\right.$ y $\left.L D_{95}\right)$ por determinaciones dosismortalidad, este fue el procedimiento adoptado y recomendado por la OMS.

En 1975, Privora (15) afirma que prefiere la determinación $\mathrm{KT}_{50}$, ya que en menor tiempo, con una cantidad pequeña de insectos y a un costo mínimo, responde con precisión si los insectos ensayados son susceptibles o no a un insecticida dado; el procedimiento de la OMS, en cambio, resultaba para el investigador más dispendioso, lento y requería de un equipo de laboratorio básico incluyendo los estuches estandarizados. Para determinar $\mathrm{KT}_{50}$, este autor empleó un procedimiento similar al propuesto por el CDC. Novakova y Jarolimova (16) siguieron la idea de Privora y determinaron resistencia de Musca domestica L a DDT a través de este método.

La idea rescatada nuevamente por los investigadores del CDC trae componentes nuevos, como es la posibilidad de ensayar mezclas de insecticidas o adicionar sinergistas para deducir el mecanismo de resistencia involucrado. En la literatura reciente sobre susceptibilidad-resistencia de especies vectores a insecticidas, ya se encuentran algunos trabajos que emplean la metodología propuesta por el CDC, como ejemplos están Hargreaves et al. (17) y Brooke et al. (18), quienes aplican este tipo de ensayos biológicos en especies de Anopheles en Africa.

En Colombia, Suárez y Morales (19) se aproximan de forma menos directa a esta técnica. En la realización de pruebas de susceptibilidad del mosquito Aedes aegypti $\mathrm{L}$ al piretroide cyflutrin aplican la metodología propuesta por la OMS, pero reemplazan los tubos estándar de exposición por botellas de vidrio de $500 \mathrm{ml}$ impregnadas con el insecticida, para impedir que los mosquitos se posen en superficies no impregnadas como puede ocurrir en los tubos de la OMS.

Una experiencia interesante de Ocampo et al. (20) evaluó la susceptibilidad a insecticidas en
Anopheles pseudopunctipennis Theobald por varios métodos, incluidos determinaciones tiempomortalidad en larvas en forma análoga al método CDC, encontrando este procedimiento sensible y práctico para ser utilizado en campo.

Este método se apoya en un concepto básico de toxicología, el cual estipula que en el momento en que un insecticida entra en contacto con el insecto, el componente debe penetrar el exoesqueleto, atravesar los tejidos intermedios, llegar al sitio de acción en el insecto y actuar sobre éste, momento en el cual el insecto evidencia la intoxicación y cae derribado. Este proceso toma una determinada cantidad de tiempo en una población susceptible. Cualquier mecanismo que el mosquito desarrolle para interferir o retrasar este proceso se considera resistencia (6).

En este estudio se realizó una aproximación a esta técnica, empleando como modelo insectos vectores de leishmaniasis. En la primera parte, se ensayó el método sin adicionar insecticida, y se obtuvieron resultados variables dependiendo de la especie. Para L. longipalpis el método resultó conveniente y los residuos de acetona en la botella no afectaron las hembras de manera importante. Para $L$. serrana y $L$. quasitownsendi, la permanencia en las botellas limpias transcurrió normalmente, mientras que en las botellas impregnadas con acetona se observaron reacciones adversas como repelencia y postración en algunas hembras.

L. longipalpis es una especie relativamente robusta en comparación con la mayoría de especies del género Lutzomyia, que son delicadas y de tamaño pequeño (21), lo que podría explicar una recuperación más rápida ante los residuos del solvente utilizado. Para las otras dos especies debe ajustarse la metodología variando factores como el solvente utilizado o el tiempo de secado.

En la determinación de una concentración diagnóstica de lambdacihalotrina para $L$. longipalpis, a una concentración de $10 \mu \mathrm{g} / \mathrm{botella}$, se obtuvo una regresión lineal que podría utilizarse como línea base para la cepa utilizada.

Según los investigadores que han rescatado y desarrollado la idea recientemente, las ventajas 
de este método frente al procedimiento convencional de la OMS son: 1) es ágil, los resultados pueden ser generados en poco tiempo; 2) las botellas pueden ser preparadas en cualquier lugar empleando cualquier insecticida grado técnico; 3) los resultados obtenidos son reproducibles sin necesidad de un alto número de insectos y réplicas, y 4) permite inferir qué mecanismo de detoxificación podría estar confiriendo resistencia (6).

Desde el punto de vista de la realización de este trabajo, otras características favorables del método son: su bajo costo, fácil uso en condiciones de campo, superficie de exposición impregnada en su totalidad y visibilidad del comportamiento de los insectos.

Es necesario continuar los estudios con este género de insectos y otros de importancia en salud pública, explorando nuevas alternativas en la determinación de resistencia a insecticidas, que agilicen la vigilancia y garanticen en este sentido el buen desempeño de las campañas de control químico.

\section{Agradecimientos}

A Richard Brogdon del CDC de Atlanta por suministrarnos el protocolo de impregnación de las botellas y la descripción de la técnica. A Marco Fidel Suárez por su colaboración en el mantenimiento de las colonias de laboratorio. Por la financiación a la Organización Panamericana de la Salud (ASC-99/00099-1), al proyecto $\mathrm{Ge}$ netics and biogeography of sand fly disease vectors (Al-34521) y al Instituto Nacional de Salud (20003100302). A Colciencias por su apoyo a grupos de investigación (convocatoria año 2000, contrato 338).

\section{Referencias}

1. Georhiou GP. The magnitude of the resistance problem in pesticide resistance: strategies and tactics for management. Washington, D.C.: National Academy Press; 1986. p.14-43.

2. World Health Organization. Vector's resistance to pesticides. Fifteenth Report of the WHO Expert Committee on Vector Biology and Control. Tech Rep Ser 818. Geneva: WHO: 1992. p.1-62.

3. Organización Mundial de la Salud. Resistencia a los insecticidas y lucha contra vectores. Serie de informes técnicos N.443. $17^{\circ}$ informe del Comité de expertos de la OMS en insecticidas. Ginebra: OMS; 1970.

4. Brogdon WG. Biochemical resistence detection: an alternative to bioassay. Parasitology Today 1989;5:56-60.

5. Shidrawi GR. A WHO global programme for monitoring vector resistance to pesticides. Bull World Health Org 1990;68:403-8.

6. Brogdon WG, McAllister J. Simplification of adult mosquito bioassays through use of time-mortality determinations in glass bottles. J Am Mosq Control Assoc 1998; 14:159-64.

7. McAllister J, Brogdon WG. The bottle bioassay for measuring resistance. Disponible en: URL: http:// www.floridamosquito.org/wing/1999/sping 99/wgbtspg 99 ew. htlm. 1999.

8. Santamaría E, Munstermann LE, Ferro C. Estandarización del método propuesto por la Organización Mundial de la Salud para determinar los niveles de susceptibilidad de los vectores de leishmaniasis a insecticidas. Biomédica 2002;22:211-8.

9. Mazzarri MB, Feliciangeli MD, Maroli M, Hernández A, Bravo A. Susceptibility of Lutzomyia longipalpis (Diptera: Psychodidae) to selected insecticides in an endemic focus of visceral leishmaniasis in Venezuela. $J$ Am Mosq Control Assoc 1997;13:335-41.

10. Seyedi MA, Yezdan H, Shah H, Jeradi M. Susceptibility of Phlebotomus papatasi (Diptera: Psychodidae) to DDT in some foci of cutaneous leishmaniasis in Iran. J Am Mosq Control Assoc 1992;8:99-100.

11. Fahmy AR, Khater EIM, EI Sawaf B, Shehata M. Insecticide susceptibility status of field population of sandfly Phlebotomus papatasi in the Sinai Peninsula, Egypt. Geneva: WHO; 1996. p.1-9.

12. Tetreault GE, El-Baset A, Hanafi AH, Beavers GM, Zeichner BC. Susceptibility of sand flies to selected insecticides in North Africa and the Middle East. J Am Mosq Control Assoc 2001;17:23-7.

13. Zerba E. Insecticidal activity of pyrethroids on insects of medical importance. Parasitology Today 1988;4:53-7.

14. Brown AW. World Health Organization. Monograph Ser. No. 38. Geneva: WHO; 1958.

15. Privora M. Use of orientative evaluation (screening) of sensitivity of flies to inseticides. J Hyg Epidemiol Microbiol Inmunol 1975;19:184-94.

16. Novakova J, Jarolimova D. Study of resistance of flies to DDT on agricultural premises in the North-Moravian region. J Hyg Epidemiol Microbiol Inmunol 1975; 19:195-9.

17. Hargreaves $\mathrm{K}$, Koekemoer LL, Brooke BD, Hunt $\mathbf{R H}$, Mthembu J, Coetzee M. Anopheles funestus resistant to pyrethroid insecticides in South Africa. Med Vet Entomol 2000;14:181-9 
18. Brooke BD, Hunt RH, Coetzee M. Resistance to dieldrin + fipronil assorts with chromosome inversion $2 \mathrm{La}$ in the malaria vector Anopheles gambiae. Med Vet Entomol 2000;14:190-4.

19. Suárez MF, Morales CA. Pruebas de susceptibilidad de Aedes aegypti a cyflutrin en Colombia. Salud Pública 2000;15:109-13.

20. Ocampo CG, Brogdon WG, Orrego CM, Toro G, Montoya-Lerma J. Insecticide susceptibility in
Anopheles pseudopunctipennis from Colombia: comparison between bioassays and biochemical assays. J Am Mosq Control Assoc 2000;16:331-8.

21. Killick-Kendrick R, Leaney AJ, Ready PD. The establishment, maintenance and productivity of a laboratory colony of Lutzomyia longipalpis (Diptera:Psychodidae). J Med Entomol 1977;3:429-40. 